\title{
Genetic differentiation in Gammarus fossarum and G. caparti (Crustacea, Amphipoda) with reference to $G$. pulex pulex in northwestern Europe
}

\author{
Maarten Scheepmaker \& Jan van Dalfsen \\ Institute of Taxonomic Zoology, University of Amsterdam, P.O. Box 4766, 1009 AT Amsterdam, The \\ Netherlands
}

Keywords: Taxonomy, distribution, morphology, genetic variability, Gammarus

\begin{abstract}
Genetic differentiation among $G$. fossarum Koch, 1835 from different stations in Germany, Switzerland, Belgium and northern France, and the closely related Belgian form $G$. caparti Pètre-Stroobants, 1980 was investigated by electrophoresis at 20 enzyme loci. Although morphologically variable, geographically distant populations of $G$. cf. fossarum were hitherto considered conspecific. In the present study, populations of $G$. cf. fossarum and $G$. caparti were examined with reference to $G$. pulex pulex as an estimate for genetic differentiation at the species level.

With $G$. p. pulex as a standard, genetic differentiation among geographically distant populations of $G$. cf. fossarum is occasionally observed at species level. The populations of $G$. caparti studied were shown to be genetically very similar to certain populations of $G$. fossarum. The taxonomic status of $G$. caparti and the genetically distinct forms of $G$. fossarum is discussed.
\end{abstract}

\section{Résumé}

La différenciation génétique de $G$. fossarum Koch, 1835, provenant de divers stations d'Allemagne, de Suisse, de Belgique et du nord de la France, et de $G$. caparti Pètre-Stroobants, 1980 (forme étroitement apparentée provenant de Belgique) a été étudiée par électrophorèse à 20 loci d'enzymes. Bien que morphologiquement variables, des populations géographiquement éloignées de $G$. cf. fossarum ont été considérées jusqu'à présent comme conspécifiques. Dans la présente étude, des populations de $\boldsymbol{G}$. cf. fossarum et $\boldsymbol{G}$. caparti ont été étudié par rapport à $\boldsymbol{G}$. pulex pulex, utilisé comme estimation de la différenciation génétique au niveau spécifique.

Avec $G$. p. pulex comme référence, la différenciation génétique entre des populations géographiquement éloignées de $G$. cf. fossarum atteint occasionnellement le niveau spécifique. Les populations de $G$. caparti étudiées se sont montrées génétique- ment très semblables à certaines populations de G. fossarum. Le statut taxonomique de $G$. caparti et celui des formes génétiquement distinctes de $G$. fossarum est discuté.

\section{Introduction}

Local populations of Gammarus fossarum Koch, 1836 have often been described as separate species (Karaman \& Pinkster, 1977; Pètre-Stroobants, $1980,1981)$. The species is widely distributed in northwestern Europe (Karaman \& Pinkster, 1977), and exhibits morphologically significant geographic variation (Goedmakers, 1972). Cross-breeding experiments among samples of morphologically distinct populations of the G. pulex-group (Pinkster, 1983; Scheepmaker, 1987) indicate complete interfertility and genetic homogeneity and these populations are therefore considered conspecific. Similarly, cross-breeding experiments among morphologically distinct allopatric samples of $G$. fossarum (Goedmakers, 1972; Scheepmaker, unpubl. data) and experiments run by Scheepmaker (unpubl. data), between $G$. caparti and $G$. fossarum also suggest complete interfertility and thus conspecificity.

The present study aims to provide an estimate of intra-specific genetic differentiation and a genetic base for the reconsideration of the conspecificity of allopatric populations of $G$. fossarum. Secondly, the taxonomic status of $G$. caparti is treated. Assessment of the species level of genetic differentia- 
Table 1. Sampling localities and species distribution.

\begin{tabular}{|c|c|c|c|c|c|c|c|}
\hline $\begin{array}{r}\text { Station } \\
\text { no. }\end{array}$ & $\begin{array}{l}\text { Area in } \\
\text { fig. } 1 \mathrm{~A}\end{array}$ & Species & Country & Prov/dept & $\begin{array}{l}\text { Drainage } \\
\text { system }\end{array}$ & $\begin{array}{l}\text { Locality } \\
\text { description }\end{array}$ & $\begin{array}{r}\text { Sampling } \\
\text { date }\end{array}$ \\
\hline 1 & D & G. fossarum & Germany & Regensburg & Donau & $\begin{array}{l}\text { Brook along road between Thalmassing and Dünzling, } \\
4 \mathrm{~km} \mathrm{W.} \mathrm{of} \mathrm{road} 15\end{array}$ & $15-V-87$ \\
\hline 2 & D & G. fossarum & Germany & Regensburg & Donau & $\begin{array}{l}\text { Brook draining in Gr. Laaber at Pinkofen, } 3 \mathrm{~km} \text { E. of } \\
\text { road } 15\end{array}$ & $15-V-87$ \\
\hline 3 & $\mathbf{E}$ & G. fossarum & Germany & Regensburg & Donau & $\begin{array}{l}\text { Brook at Wutzdorf, } 1 \mathrm{~km} \mathrm{~S} \text {. of road } 20,24 \mathrm{~km} \mathrm{N.N.E.} \\
\text { of Regensburg }\end{array}$ & $15-V-87$ \\
\hline 4 & $\mathbf{F}$ & G. fossarum & Switzerland & Oberaargau & Langete & $\begin{array}{l}\text { Stream draining in Langete at Kleindietwill, } 3 \mathrm{~km} \mathrm{~N} \text {. E. } \\
\text { of road } 23\end{array}$ & 17-V-87 \\
\hline 5 & $\mathbf{F}$ & $\begin{array}{l}\text { G. fossarum } \\
\text { G.p.pulex }\end{array}$ & Switzerland & Bem & Aare & $\begin{array}{l}\text { Stream draining into Aare, at Oberthal, } 4 \mathrm{~km} \mathrm{~N} \text {. of } \\
\text { road } 30\end{array}$ & $17-V-87$ \\
\hline 6 & $\mathbf{F}$ & G. fossarum & Switzerland & Bern & Aare & $\begin{array}{l}\text { Brook drainig into Aare, near Frieswill, } 8 \mathrm{~km} \mathrm{~S} \text {. E. of } \\
\text { road } 22 \text { at Aarberg }\end{array}$ & $17-V-87$ \\
\hline 7 & C & G. caparti & Belgium & Luxembourg & Meuse & $\begin{array}{l}\text { Lomme, at the Mirwart fish farm, } 1 \mathrm{~km} \mathrm{S.} \mathrm{E.} \mathrm{of} \mathrm{road} \\
\text { N46 }\end{array}$ & 20-VI-87 \\
\hline 8 & C & G. caparti & Belgium & Liège & Meuse & $\begin{array}{l}\text { Samson, at the crossing with road } \mathrm{N} 42,1 \mathrm{~km} \mathrm{~N} \text {. of } \\
\text { Faulx-les-Tombes }\end{array}$ & 20 -VI-87 \\
\hline 9 & C & $\begin{array}{l}\text { G. fossarum } \\
\text { G.p.pulex }\end{array}$ & Belgium & Namur & Escaut & $\begin{array}{l}\text { Source/brooklet draining into the Dyle, near N21, } \\
3.5 \mathrm{~km} \mathrm{~N} \text {. W. of Corroy-le-Chateau }\end{array}$ & \\
\hline 10 & C & G. fossarum & Belgium & Brabant & Lzer & $\begin{array}{l}\text { Rivulet in woodland along road N75 draining into the } \\
\text { Kemmelbeek, } 2 \mathrm{~km} \mathrm{S.} \mathrm{E.} \mathrm{of} \mathrm{Westouter}\end{array}$ & 22-VI-'87 \\
\hline 11 & B & G. fossarum & France & Pas-de-Calais & Liane & $\begin{array}{l}\text { Brooklet in the Forret de Boulogne draining into the } \\
\text { Liane, near road D254, } 9 \mathrm{~km} \text { E. of Boulogne-sur-Mer }\end{array}$ & 21-VI-'87 \\
\hline 12 & B & G. fossarum. & France & Pas-de-Calais & Wimereux & $\begin{array}{l}\text { Upper reach of the Wimereux, along road } 251 \mathrm{e}, 1 \mathrm{~km} \\
\text { S. S. E. of Boursin }\end{array}$ & 21-VI-'87 \\
\hline 13 & B & G. fossarum & France & Pas-de-Calais & Slack & $\begin{array}{l}\text { Country road at Héronval, near road D127, } 3 \mathrm{~km} \mathrm{S.} \mathrm{E.} \mathrm{E.} \\
\text { of Réty }\end{array}$ & 21-VI-'87 \\
\hline 14 & B & $\begin{array}{l}\text { G. fossarum } \\
\text { G.p.pulex }\end{array}$ & France & Pas -de-Calias & Slack & $\begin{array}{l}\text { Upper reach/source of the Slack, along road 25le, } \\
2.5 \mathrm{~km} \mathrm{~N} \text {. E. of Boursin }\end{array}$ & 21-VI-'87 \\
\hline 15 & - & G. p.pulex & Holland & Gelderland & $\begin{array}{l}\text { Hierdense } \\
\text { beek }\end{array}$ & $\begin{array}{l}\text { Hulshorst, } 0.2 \mathrm{~km} \mathrm{~N} \text {. N. W. of the railroad station } \\
\text { and road E35 }\end{array}$ & 2-I-'88 \\
\hline
\end{tabular}

tion is based on 4 geographic samples of $G$. pulex pulex (Linnaeus, 1758). G. p. pulex is morphologically well-defined, often found sympatric with $G$. fossarum, but maintains its integrity in these areas (Wautier \& Roux, 1959; Roux, 1971; Meijering, 1972).

In this study of samples from northwestern Europe, genetic variation is investigated using starch gel enzyme electrophoresis.

\section{Materials and methods}

\section{Sampling and collection sites}

Sampling for electrophoretic studies has been carried out based on the procedures of Scheepmaker, 1987. The collection sites and sampling dates along with the species collected are listed in table 1 and fig. 1A-F. Four sampling areas in northern
France, Belgium, Switzerland, and southern Germany were selected as exhibiting extremes in morphology. The morphological variation recorded (following criteria of Goedmakers, 1972) is summarized in table 2 and fig. 2. G. caparti (table 1, fig. 1A, C: stations 7,8 ) differs from $G$. fossarum primarily by the presence of a seta on the first segment of the right mandibular palp in most (but not all) individuals (fig. 2E). G. fossarum from stations 10-14 in northern France and Belgium (table 1; fig. 1A, B and C) differs from individuals of all other populations by the short setation of the $A 2$ and $P 4$ (fig. 2B, G). Individuals from Swiss populations of G. fossarum (table 1; fig. 1A, F) are characterized by much longer setae on the flagellum (fig. 2A). The presence or absence of calceoli (fig. 2B) is usually constant within populations. In German populations this character can be variable; in populations of $G$. fossarum from the Regensburg area 

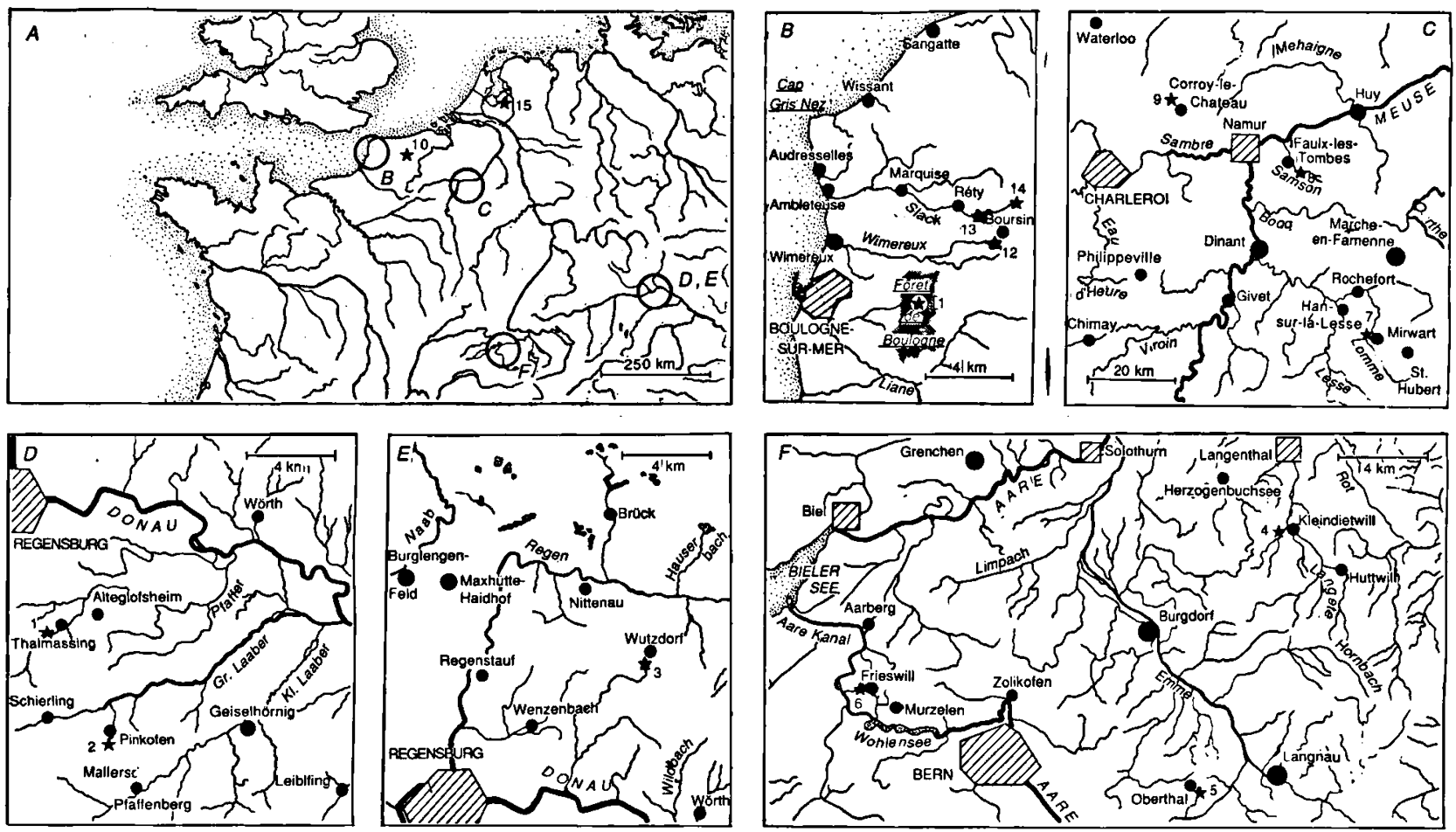

Fig. IA-F. Study areas and sampling localities (cf. table 1).

Table 2. Morphological differentiation of some characters in $G$. fossarum and $G$. caparti. For legend see fig. 2.

Station A2: Length of the setae with reference

no to the diameter of the segments

on which they are inplanted
Density setation

Setation Calccoli seta on

P3 present first

curly segment

mandibular

palp

\begin{tabular}{cccccccccc}
\hline $\begin{array}{c}\text { peduncle } \\
\text { segment }\end{array}$ & $\begin{array}{l}\text { flagel- } \\
\text { lum }\end{array}$ & & & & & & & & \\
\hline 4 & 5 & & P3 & P4 & A2 & P3 & P4 & U3 \\
\hline
\end{tabular}

\begin{tabular}{|c|c|c|c|c|c|c|c|c|c|c|c|c|}
\hline 1 & $=$ & $>=$ & $=$ & $\gg$ & $=$ & - & ++ & + & ++ & + & $-1+$ & - \\
\hline 2 & $=$ & $>=$ & $=$ & $\gg$ & $=$ & - & ++ & + & ++ & + & $-1+$ & - \\
\hline 3 & $=$ & $>=$ & $=$ & $\gg$ & $>=$ & • & ++ & + & + & + & $-1+$ & - \\
\hline 4 & $=$ & $>=$ & $\gg=$ & $\gg$ & $>=$ & + & ++ & + & + & - & + & - \\
\hline 5 & $>=$ & $>>=$ & $\gg=$ & $>$ & $>=$ & + & + & + & + & + & + & - \\
\hline 6 & $=$ & $>=$ & $\gg=$ & $\gg$ & $>=$ & + & ++ & + & - & + & + & - \\
\hline 7 & $=$ & $>=$ & $=$ & $\gg$ & $>=$ & $-1+$ & + & - & + & - & + & $-1+$ \\
\hline 8 & $=$ & $>=$ & $=$ & $\gg$ & $>=$ & $-1+$ & + & - & + & - & + & $-1+$ \\
\hline 9 & $<$ & $<$ & $<$ & $\gg$ & $>$ & $-1+$ & ++ & + & ++ & + & + & - \\
\hline 10 & $<=$ & $<$ & $\ll$ & $\gg$ & $=$ & $\cdot$ & ++ & - & + & + & + & $\cdot$ \\
\hline 11 & $<=$ & $<$ & $<$ & $>$ & $=$ & - & + & - & + & + & + & - \\
\hline 12 & $<=$ & $<=$ & $<$ & $>$ & $=$ & - & - & - & $-1+$ & - & + & - \\
\hline 13 & $<=$ & $<$ & $<$ & $>$ & $\Leftrightarrow$ & - & + & - & $-1+$ & - & + & - \\
\hline 14 & $<=$ & $<$ & $<$ & $>$ & $=$ & - & + & • & $-1+$ & - & + & - \\
\hline
\end{tabular}




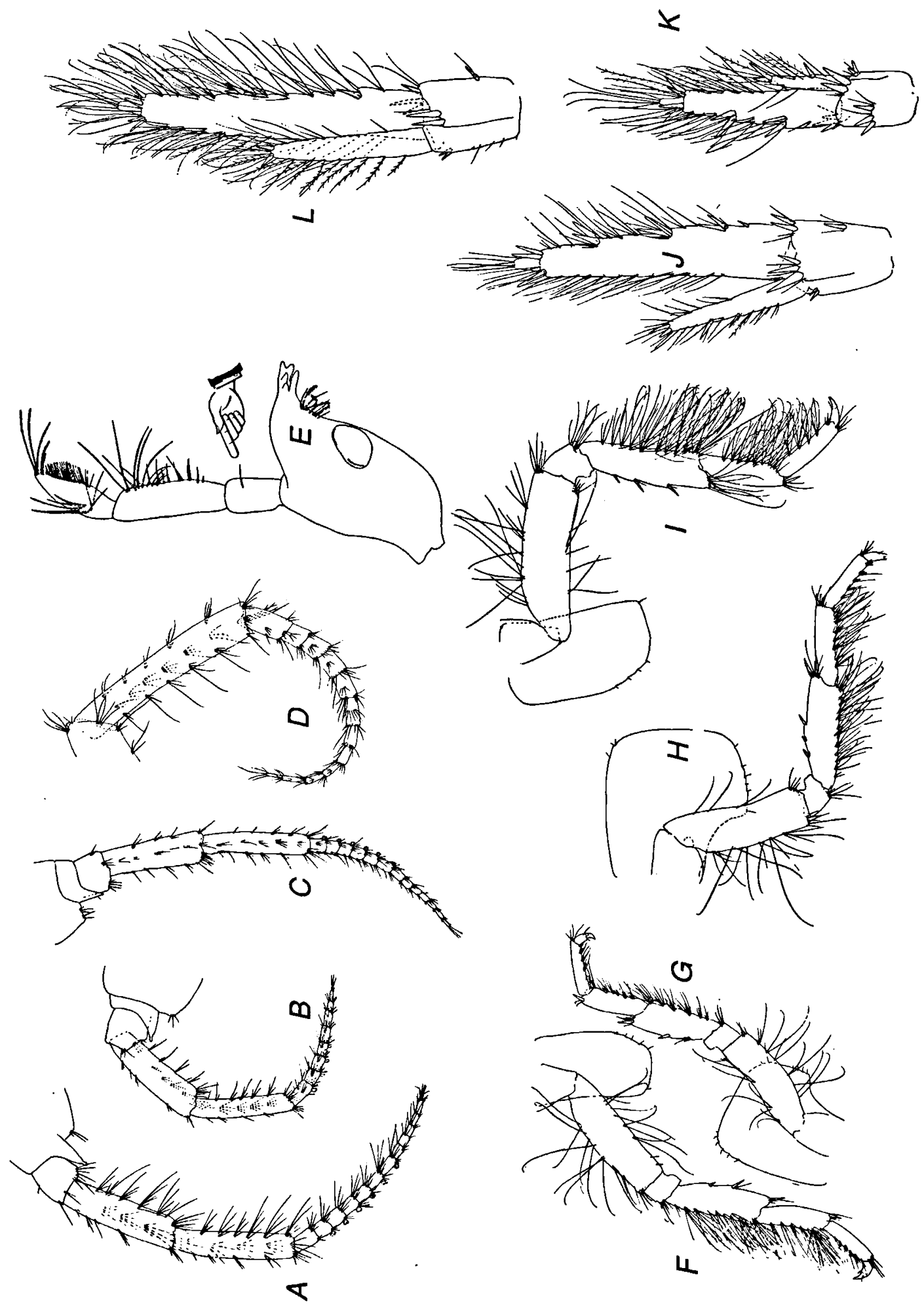


some individuals lack calceoli. However, the variation of most morphological characters overlaps among the populations studied. In each area 3 samples were taken to evaluate local genetic variation. The sampling localities in southern Germany are from the surrounding area of the former type locality of $G$. fossarum in the Regensburg area. The sampling localities in Belgium include the type locality of $G$. caparti (station 7); however, at 2 previously recorded sampling localities of $G$. caparti (Pètre-Stroobants, 1980) only $G$. fossarum was found (stations 9,10 ).

\section{Electrophoresis}

Electrophoresis and staining procedures were identical to those of Scheepmaker et al. (1988). The following enzyme systems were assayed: ADA Adenosine deaminase, E.C. No. 3.5.4.4; ALP Alkaline phosphatase, E.C. No. 3.1.3.1; APK Arginine phosphate kinase, E.C. No. 2.7.3.3; EST - Esterase, E.C. No. 3.1.1.1; GDH - Glutamate dehydrogenase, E.C. No. 1.1.1.47; GOT - Glutamic oxaloacetic transaminase, E.C. No. 2.6.1.1;
GPI - Glucose phosphate isomerase, E.C. No. 5.3.1.11; HK - Hexokinase, E.C. No. 2.7.1.1; LAP - Leucine aminopeptidase, E.C. No. 3.4.1.1; MDH - Malate dehydrogenase, E.C. No. 1.1.1.37; ME - Malic enzyme, E.C. No. 1.1.1.40; MPI Mannosephosphate isomerase, E.C. No. 5.3.1.8; PEP - Peptidase, E.C. No. 3.4.11/13; 6PGD - 6 Phosphogluconate dehydrogenase, E.C. No. 1.1.1.44; PK - Pyruvate kinase, E.C. No. 2.7.1.40.

\section{Analysis of allozyme variation}

The genetic interpretation of the variation was inferential. Electromorph frequencies and matrices of genetic identity $(I)$ and $(D)$ according to Nei (1972) and Rogers' (1972) genetic distance were calculated with the computer program BIOSYS-1 (Swofford \& Selander, 1981). From these data, an UPGMA dendrogram (Sneath \& Sokal, 1973) and a distance Wagner network (Farris, 1972) were constructed.

Fig. 2. Morphological differentiation of selected variable characters in G. fossarum and G. caparti. In parentheses: symbols used in table 2.

A: A2, bearing calceoli, setation of peduncle segments 4,5 and flagellum much longer than or equal to the diameter of the segments on which they are implanted $(>>=)$;

B: A2, bearing calceoli, setation of peduncle segments 4,5 and flagellum predominantly equal to or shorter than the diameter of the segments on which they are implanted $(<=)$;

C: A2, bearing calceoli, setation of the peduncle segments 4,5 and flagellum much shorter than the diameter of the segments on which they are implanted $(<)$;

D: A2, without calceoli, setation of the peduncle segments 4,5 and flagellum equal to or longer than the diameter of the segments on which they are implanted $(>=)$;

E: right mandibular palp of $G$. caparti with a seta implanted on the first segment;

F: P3, setae longer than or equal to the diameter of the segment on which they are implanted, setosity moderate (") = " and " + ", respectively), setae not curly;

G: P4, setae shorter than or equal to the diameter of the segment on which they are implanted, setosity poor (" < =" and " - ", respectively);

H: idem, setae longer than or equal to the diameter of the segment on which they are implanted, setosity moderate (" $>$ = " and " + ", respectively);

I: P3, setae much longer than the diameter of the segment on which they are implanted, setosity dense (" $>>$ " and " + + ", respectively), setae curly;

J: Uropod 3, poor setation (" - ");

$\mathrm{K}$ : idem, moderate setation (“+");

L: idem, rich setation (" ++ ");

(Drawings from: Goedmakers, 1972; Karaman \& Pinkster, 1977; Pètre-Stroobants, 1980). 
Table 3. Electromorph frequencies at $\mathbf{1 3}$ presumptive gene loci.

$\begin{array}{lllllllllllllllllll}\text { station } & 1 & 2 & 3 & 4 & 5 & 6 & 7 & 8 & 9 & 10 & 11 & 12 & 13 & 14 & 5 & 9 & 14 & 15 \\ & f & f & f & f & f & f & c & c & f & f & f & f & f & f & p & p & p & p\end{array}$

locus

electromorph

$\begin{array}{llllllllllllllllllll}A p k & (\mathrm{~N}) & 38 * & 35 & 66 & 66 & 41 & 50 & 40 & 43 & 37 & 43 & 39 & 35 & 43 & 22 & 6 & 35 & 28 & 30\end{array}$

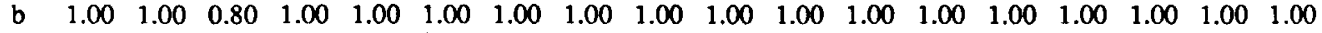

$\begin{array}{llllllllllllllllllll}\text { c } & 0.00 & 0.00 & 0.20 & 0.00 & 0.00 & 0.00 & 0.00 & 0.00 & 0.00 & 0.00 & 0.00 & 0.00 & 0.00 & 0.00 & 0.00 & 0.00 & 0.00 & 0.00\end{array}$

$\begin{array}{llllllllllllllllllll}\text { Est-1 } & (\mathrm{N}) & 40 & 47 & 44 & 29 & 35 & 52 & 42 & 61 & 37 & 29 & 27 & 44 & 29 & 19 & 3 & 26 & 21 & 20\end{array}$

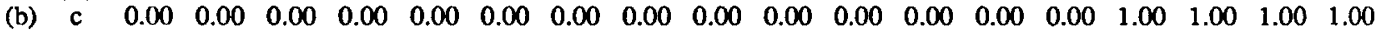

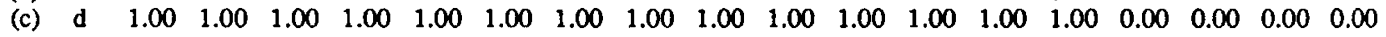

$\begin{array}{llllllllllllllllllll}\text { Got-1 } & (\mathrm{N}) & 41 & 62 & 70 & 68 * & 39 & 59 & 56 & 30 & 39 & 39 & 33 & 33 & 36 & 21 & 3 & 35 & 50 & 33\end{array}$

$\begin{array}{ccccccccccccccccccc}\text { a } & 0.79 & 0.88 & 0.82 & 0.46 & 1.00 & 0.23 & 0.04 & 0.00 & 0.00 & 0.00 & 0.00 & 0.00 & 0.00 & 0.00 & 0.00 & 0.00 & 0.00 & 0.00\end{array}$

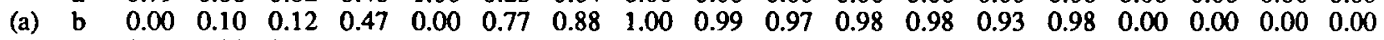

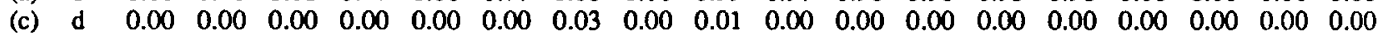

$\begin{array}{lllllllllllllllllll}\text { e } & 0.00 & 0.00 & 0.01 & 0.00 & 0.00 & 0.00 & 0.00 & 0.00 & 0.00 & 0.00 & 0.00 & 0.00 & 0.00 & 0.00 & 0.00 & 0.00 & 0.00 & 0.00\end{array}$

f $\quad \begin{array}{llllllllllllllllllll} & 0.00 & 0.01 & 0.00 & 0.00 & 0.00 & 0.00 & 0.05 & 0.00 & 0.00 & 0.03 & 0.02 & 0.02 & 0.07 & 0.02 & 1.00 & 1.00 & 1.00 & 1.00\end{array}$

$\begin{array}{lllllllllllllllllll}\text { g } & 0.21 & 0.01 & 0.05 & 0.07 & 0.00 & 0.00 & 0.00 & 0.00 & 0.00 & 0.00 & 0.00 & 0.00 & 0.00 & 0.00 & 0.00 & 0.00 & 0.00 & 0.00\end{array}$

$\begin{array}{lllllllllllllllllllll}h & 0.31 & 0.24 & 0.12 & 0.26 & \bullet & 0.19 & 0.20 & \bullet & 0.03 & 0.05 & 0.03 & 0.03 & 0.12 & 0.05\end{array}$

$\begin{array}{lllllllllllllllllllll}\text { Got-2 } & (\mathrm{N}) & 39 & 62 & 69 & 56 & 36 & 48 & 56 & 30 & 39 & 39 & 33 & 33 & 36 & 21 & 3 & 35 & 50 & 33\end{array}$

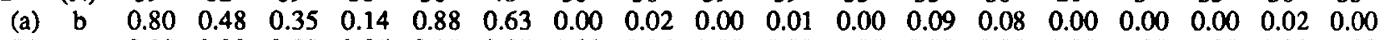

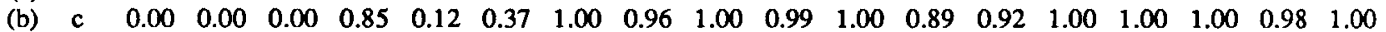

$\begin{array}{llllllllllllllllllll}\text { (c) } & d & 0.20 & 0.52 & 0.65 & 0.01 & 0.00 & 0.00 & 0.00 & 0.02 & 0.00 & 0.00 & 0.00 & 0.02 & 0.00 & 0.00 & 0.00 & 0.00 & 0.00 & 0.00\end{array}$

$\begin{array}{lllllllllllllllllllll}h & 0.26 & 0.42 & 0.44 & 0.16 & 0.03 & 0.33 & - & 0.70 & 0.02 & 0.03 & - & 0.15 & 0.11 & - & - & - & 0.04 & \end{array}$

$\begin{array}{llllllllllllllllllll}G p i & (\mathrm{~N}) & 76 & 107 & 59 & 80 & 20 & 80 & 61 & 54 & 52 & 51 & 30 & 53 & 45 & 45 & 9 & 40 & 32 & 37\end{array}$ a $\quad \begin{array}{llllllllllllllllll}0.00 & 0.00 & 0.00 & 0.00 & 0.00 & 0.00 & 0.00 & 0.00 & 0.00 & 0.56 & 1.00 & 0.99 & 0.96 & 0.98 & 0.00 & 0.00 & 0.00 & 0.00\end{array}$

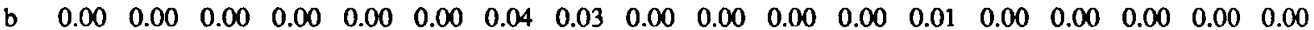

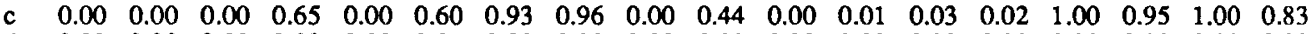

$\begin{array}{llllllllllllllllllll}\text { d } & 0.00 & 0.00 & 0.00 & 0.03 & 0.00 & 0.01 & 0.00 & 0.00 & 0.00 & 0.00 & 0.00 & 0.00 & 0.00 & 0.00 & 0.00 & 0.00 & 0.00 & 0.00\end{array}$

(d) $\quad$ e $\quad \begin{array}{lllllllllllllllllll} & 0.99 & 0.76 & 0.56 & 0.18 & 0.97 & 0.37 & 0.03 & 0.01 & 0.99 & 0.00 & 0.00 & 0.00 & 0.00 & 0.00 & 0.00 & 0.00 & 0.00 & 0.14\end{array}$

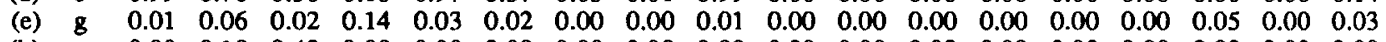

(h) $\quad \mathrm{m} \quad \begin{array}{lllllllllllllllllll} & 0.00 & 0.10 & 0.42 & 0.00 & 0.00 & 0.00 & 0.00 & 0.00 & 0.00 & 0.00 & 0.00 & 0.00 & 0.00 & 0.00 & 0.00 & 0.00 & 0.00 & 0.00\end{array}$ $\begin{array}{llllllllllllllllll}0.00 & 0.08 & 0.00 & 0.00 & 0.00 & 0.00 & 0.00 & 0.00 & 0.00 & 0.00 & 0.00 & 0.00 & 0.00 & 0.00 & 0.00 & 0.00 & 0.00 & 0.00\end{array}$ $\begin{array}{lllllllllllllllllllllll}0.26 & 0.35 & 0.50 & 0.53 & 0.05 & 0.39 & 0.13 & 0.07 & 0.02 & 0.41 & - & 0.02 & 0.09 & 0.04 & \bullet & 0.10 & \bullet & 0.27\end{array}$

$\begin{array}{llllllllllllllllllll}I / k & (\mathrm{~N}) & 34 & 53 & 63 & 36 & 39 & 39 & 37 & 40 & 35 & 36 & 34 & 37 & 36 & 15 & 39 & 35 & 25 & 16\end{array}$ $\begin{array}{lllllllllllllllllll}\text { a } & 1.00 & 1.00 & 1.00 & 0.00 & 0.00 & 0.00 & 0.00 & 0.00 & 0.00 & 0.00 & 0.00 & 0.00 & 0.00 & 0.00 & 0.00 & 0.00 & 0.00 & 0.00\end{array}$ $\begin{array}{lllllllllllllllllll}\text { b } & 0.00 & 0.00 & 0.00 & 1.00 & 1.00 & 1.00 & 1.00 & 1.00 & 1.00 & 1.00 & 1.00 & 1.00 & 1.00 & 1.00 & 1.00 & 1.00 & 1.00 & 1.00\end{array}$

$\begin{array}{cccccccccccccccccccc}M d h-1 & \text { (N) } & 41 & 61 & 76 & 70 & 28 & 64 & 58 & 30 & 39 & 41 & 33 & 33 & 46 & 32 & 3 & 34 & 38 & 19\end{array}$

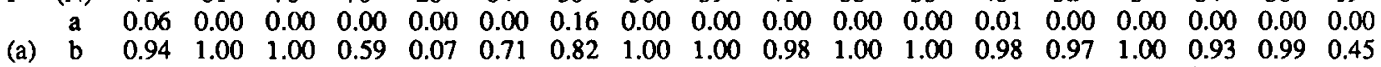

$\begin{array}{lllllllllllllllllll}\mathrm{d} & 0.00 & 0.00 & 0.00 & 0.00 & 0.00 & 0.00 & 0.00 & 0.00 & 0.00 & 0.00 & 0.00 & 0.00 & 0.00 & 0.00 & 0.00 & 0.07 & 0.00 & 0.00\end{array}$

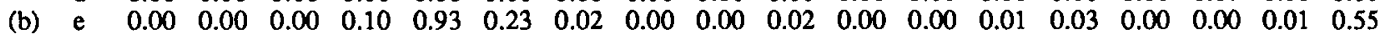

(c) f $\quad \begin{array}{llllllllllllllllllll} & 0.00 & 0.00 & 0.00 & 0.30 & 0.00 & 0.06 & 0.00 & 0.00 & 0.00 & 0.00 & 0.00 & 0.00 & 0.00 & 0.00 & 0.00 & 0.00 & 0.00 & 0.00\end{array}$

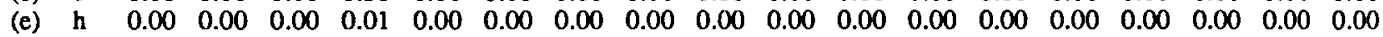

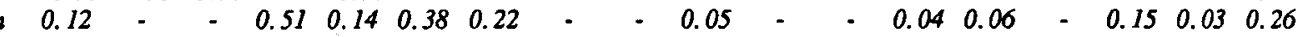

$\begin{array}{llllllllllllllllllll}M d h-2 & (\mathrm{~N}) & 34 & 57 & 76 & 66 & 28 & 62 & 58 & 29 & 39 & 30 & 33 & 27 & 41 & 32 & 3 & 34 & 38 & 33\end{array}$ $\begin{array}{ccccccccccccccccccc}\mathrm{b} & 0.00 & 0.02 & 0.00 & 0.00 & 0.00 & 0.00 & 0.00 & 0.00 & 0.00 & 0.00 & 0.00 & 0.00 & 0.00 & 0.00 & 0.00 & 0.00 & 0.00 & 0.00\end{array}$ $\begin{array}{llllllllllllllllllll}\text { c } & 1.00 & 0.98 & 1.00 & 1.00 & 1.00 & 1.00 & 0.98 & 1.00 & 1.00 & 1.00 & 1.00 & 1.00 & 1.00 & 1.00 & 1.00 & 1.00 & 1.00 & 1.00\end{array}$ $\begin{array}{lllllllllllllllllll}\text { d } & 0.00 & 0.00 & 0.00 & 0.00 & 0.00 & 0.00 & 0.02 & 0.00 & 0.00 & 0.00 & 0.00 & 0.00 & 0.00 & 0.00 & 0.00 & 0.00 & 0.00 & 0.00\end{array}$

h $\quad-0.03 \quad-\quad-\quad-\quad-0.03$

$\begin{array}{llllllllllllllllllll}M e & (\mathrm{~N}) & 36 & 78 & 67 & 54 & 43 & 60 & 45 & 49 & 51 & 46 & 42 & 36 & 46 & 14 & 3 & 43 & 20 & 11\end{array}$ $\begin{array}{lllllllllllllllllll}\text { b } & 1.00 & 1.00 & 1.00 & 0.00 & 1.00 & 0.00 & 0.00 & 0.00 & 1.00 & 0.00 & 0.00 & 0.00 & 0.00 & 0.00 & 0.00 & 0.00 & 0.00 & 0.00\end{array}$ $\begin{array}{llllllllllllllllllll}\text { c } & 0.00 & 0.00 & 0.00 & 1.00 & 0.00 & 1.00 & 1.00 & 1.00 & 0.00 & 1.00 & 1.00 & 1.00 & 1.00 & 1.00 & 1.00 & 1.00 & 1.00 & 1.00\end{array}$ 
Table 3. Continued.

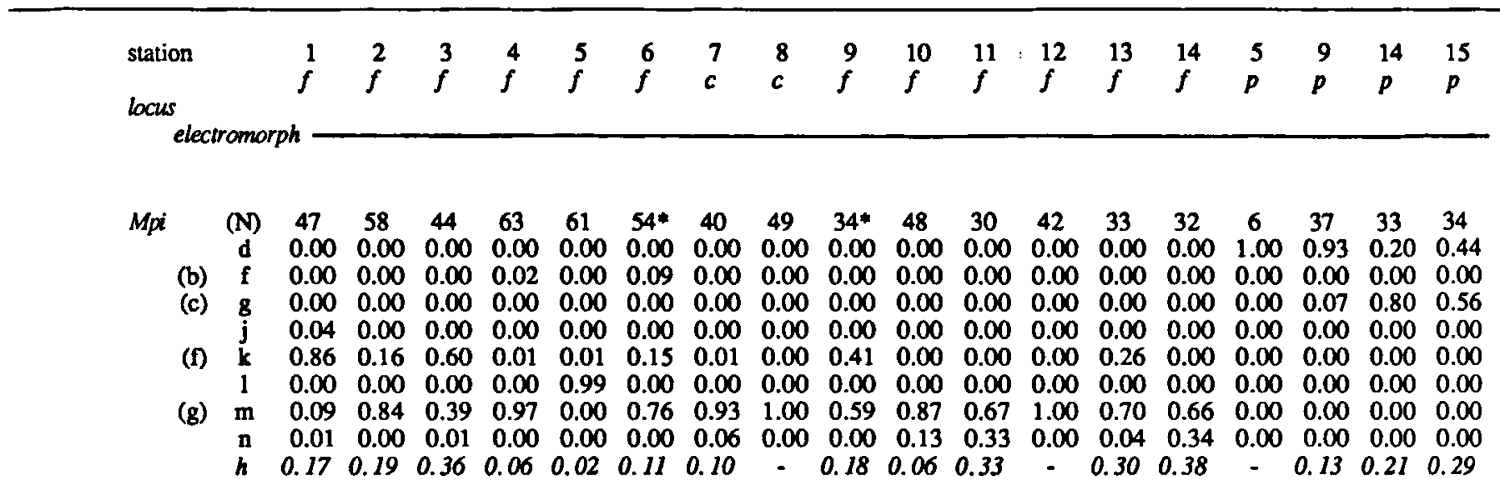

$\begin{array}{llllllllllllllllllll}\text { Pep-2 } & \text { (N) } & 43^{*} & 46 * & 32 & 33 & 42 & 40 & 59 & 41 & 46 & 41 & 34 & 29 & 53 & 10 & 5 & 47 & 30 & 26\end{array}$

$\begin{array}{ccccccccccccccccccc}\text { c } & 0.00 & 0.00 & 0.00 & 0.00 & 0.00 & 0.00 & 0.00 & 0.00 & 0.00 & 0.00 & 0.00 & 0.00 & 0.00 & 0.00 & 1.00 & 1.00 & 0.95 & 1.00\end{array}$

(e) f $\quad \begin{array}{lllllllllllllllllllll} & 0.45 & 0.46 & 0.70 & 0.00 & 0.00 & 0.00 & 0.00 & 0.00 & 0.67 & 0.00 & 0.00 & 0.00 & 0.00 & 0.00 & 0.00 & 0.00 & 0.02 & 0.00\end{array}$ $\begin{array}{lllllllllllllllllll}\text { h } & 0.51 & 0.53 & 0.27 & 0.00 & 1.00 & 0.00 & 0.00 & 0.00 & 0.33 & 0.01 & 0.00 & 0.00 & 0.00 & 0.05 & 0.00 & 0.00 & 0.03 & 0.00\end{array}$ $\begin{array}{lllllllllllllllllllll}\text { i } & 0.04 & 0.00 & 0.00 & 0.00 & 0.00 & 0.00 & 0.00 & 0.00 & 0.00 & 0.00 & 0.00 & 0.00 & 0.00 & 0.00 & 0.00 & 0.00 & 0.00 & 0.00\end{array}$ $\begin{array}{lllllllllllllllllll}\text { j } & 0.00 & 0.01 & 0.03 & 1.00 & 0.00 & 1.00 & 1.00 & 1.00 & 0.00 & 0.99 & 1.00 & 1.00 & 1.00 & 0.95 & 0.00 & 0.00 & 0.00 & 0.00\end{array}$

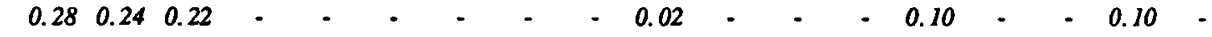

$\begin{array}{rccccccccccccccccccc}\text { Pep-4 } & \text { (N) } & 41 & 43 & 42 & 41 & 43 & 45 & 57 & 42 & 45 & 41 & 36 & 29 & 53 & 10 & 5 & 48 & 30 & 26 \\ & \text { a } & 0.00 & 0.00 & 0.00 & 0.01 & 0.00 & 0.07 & 0.56 & 0.02 & 0.00 & 0.00 & 0.01 & 0.00 & 0.00 & 0.00 & 0.00 & 0.00 & 0.00 & 0.00 \\ & \text { b } & 0.00 & 0.00 & 0.00 & 0.00 & 0.00 & 0.00 & 0.00 & 0.00 & 0.00 & 0.00 & 0.00 & 0.00 & 0.00 & 0.00 & 0.00 & 0.46 & 0.00 & 0.00 \\ \text { (a) } & \text { c } & 0.00 & 0.00 & 0.00 & 0.95 & 0.00 & 0.82 & 0.39 & 0.98 & 1.00 & 1.00 & 0.99 & 1.00 & 1.00 & 1.00 & 1.00 & 0.53 & 1.00 & 1.00 \\ & \text { e } & 0.99 & 1.00 & 1.00 & 0.00 & 0.00 & 0.00 & 0.05 & 0.00 & 0.00 & 0.00 & 0.00 & 0.00 & 0.00 & 0.00 & 0.00 & 0.01 & 0.00 & 0.00 \\ & f & 0.01 & 0.00 & 0.00 & 0.04 & 0.00 & 0.02 & 0.00 & 0.00 & 0.00 & 0.00 & 0.00 & 0.00 & 0.00 & 0.00 & 0.00 & 0.00 & 0.00 & 0.00 \\ & \text { g } & 0.00 & 0.00 & 0.00 & 0.00 & 0.00 & 0.09 & 0.00 & 0.00 & 0.00 & 0.00 & 0.00 & 0.00 & 0.00 & 0.00 & 0.00 & 0.00 & 0.00 & 0.00 \\ & h & 0.02 & - & - & 0.10 & - & 0.27 & 0.32 & 0.05 & - & - & 0.03 & - & - & - & - & 0.40 & - & -\end{array}$

$\begin{array}{llllllllllllllllllll}P k & \text { (N) } & 40 & 61 & 63 & 65 & 41 & 70 & 45 & 40 & 47 & 45 & 35 & 35 & 45 & 15 & 2 & 40 & 35 & 31\end{array}$ $\begin{array}{llllllllllllllllll}0.00 & 0.00 & 0.00 & 0.00 & 0.00 & 0.00 & 0.00 & 0.00 & 0.87 & 0.00 & 0.00 & 0.00 & 0.00 & 0.00 & 1.00 & 1.00 & 1.00 & 1.00\end{array}$ $\begin{array}{llllllllllllllllll}0.88 & 0.80 & 0.89 & 0.89 & 1.00 & 0.81 & 1.00 & 1.00 & 0.13 & 1.00 & 1.00 & 1.00 & 1.00 & 1.00 & 0.00 & 0.00 & 0.00 & 0.00\end{array}$ $\begin{array}{llllllllllllllllll}0.13 & 0.20 & 0.11 & 0.12 & 0.00 & 0.19 & 0.00 & 0.00 & 0.00 & 0.00 & 0.00 & 0.00 & 0.00 & 0.00 & 0.00 & 0.00 & 0.00 & 0.00\end{array}$ $\begin{array}{lllllllllllllllllll}h & 0.20 & 0.36 & 0.22 & 0.23 & - & 0.29 & - & - & 0.21 & - & - & - & - & - & - & - & - & -\end{array}$

$\begin{array}{lllllllllllllllllll}H & 0.07 & 0.03 & 0.04 & 0.04 & 0.01 & 0.33 & 0.02 & 0.01 & 0.02 & 0.03 & 0.02 & 0.01 & 0.03 & 0.03 & - & 0.02 & 0.02 & 0.04\end{array}$

$f=G$. fossarum; $c=G$. caparti; $p=G . p$. pulex; $\mathrm{N}=$ sample size; $h=$ heterozygosity per locus (direct count);

$H=$ mean heterozygosity over all (including monomorphic) loci;

* = significant departure from Hardy-Weinberg distribution $(P<0.05)$; electromorph designation from fastest to slowest in alphabetical order, in accordance with Scheepmaker (in prep.); in parentheses: alternative allelic designation employed by Scheepmaker et al., 1988.

\section{Results}

Twenty presumptive gene loci from 17 enzyme systems were scorable in all populations. Electromorph frequencies and relative mobilities of the enzymes coded for by these loci are listed in table 3.
The Ada, Alp-1, Alp-2, Est-2, Gdh, 6Pgd and Lap loci were monomorphic for all populations. The number of loci and subunit structures resolved were consistent with the interpretation of Scheepmaker et al. (1988). 
Table 4. Number of diagnostic loci (0.99 criterion; Ayala \& Powell, 1972) among samples of the populations studied.

\begin{tabular}{|c|c|c|c|c|c|c|c|c|c|c|c|c|c|c|c|c|c|c|c|}
\hline station no. & species & 1 & 2 & 3 & 4 & 5 & 6 & 7 & 8 & 9 & 10 & 11 & 12 & 13 & 14 & 5 & 9 & 14 & 15 \\
\hline 1 & G. fossarum & - & & & & & & & & & & & & & & & & & \\
\hline 2 & G. fossarum & - & - & & & & & & & & & & & & & & & & \\
\hline 3 & G. fossarum & $=$ & - & - & & & & & & & & & & & & & & & \\
\hline 4 & G. fossarum & 3 & 3 & 3 & - & & & & & & & & & & & & & & \\
\hline 5 & G. fossarum & 2 & 2 & 2 & 3 & - & & & & & & & & & & & & & \\
\hline 6 & G. fossarum & 4 & 3 & 3 & - & 4 & - & & & & & & & & & & & & \\
\hline 7 & G. coparti & 3 & 2 & 4 & - & 4 & - & - & & & & & & & & & & & \\
\hline 8 & G. caparti & 5 & 3 & 3 & - & 4 & - & - & - & & & & & & & & & & \\
\hline 9 & G. fossarum & 3 & 3 & 3 & 1 & 3 & 2 & 2 & 2 & - & & & & & & & & & \\
\hline 10 & G. fossarum & 5 & 4 & 4 & - & 5 & - & $=$ & - & 2 & - & & & & & & & & \\
\hline 11 & G. fossarum & 6 & 5 & 5 & 1 & 6 & 1 & 1 & 2 & 3 & - & - & & & & & & & \\
\hline 12 & G. fossarum & 6 & 4 & 4 & - & 6 & - & - & - & 3 & - & - & - & & & & & & \\
\hline 13 & G. fossarum & 6 & 4 & 4 & - & 5 & - & - & - & 3 & - & - & - & - & & & & & \\
\hline 14 & G. fossarum & 5 & 5 & 5 & - & 5 & - & - & - & 2 & - & - & - & - & - & & & & \\
\hline 5 & G.p.pulex & 8 & 9 & 10 & 5 & 7 & 5 & 4 & 4 & 6 & 4 & 5 & 4 & 4 & 4 & - & & & \\
\hline 9 & G.p.pulex & 5 & 7 & 7 & 5 & 7 & 5 & 3 & 6 & 5 & 4 & 5 & 4 & 4 & 4 & - & - & & \\
\hline 14 & G.p.pulex & 7 & 8 & 8 & 5 & 7 & 5 & 4 & 6 & 5 & 4 & 4 & 4 & 4 & 2 & - & - & - & \\
\hline 15 & G.p.pulex & 7 & 9 & 8 & 5 & 7 & 5 & 4 & 6 & 5 & 4 & 4 & 4 & 4 & 3 & - & 1 & - & - \\
\hline
\end{tabular}

Table 5. Matrix of similarity and distance coefficients. Below diagonal: Nei's (1972) genetic distance. Above diagonal: Nei's (1972) genetic identity.

\begin{tabular}{|c|c|c|c|c|c|c|c|c|c|c|c|c|c|c|c|c|c|c|c|}
\hline station no. & species & 1 & 2 & 3 & 4 & 5 & 6 & 7 & 8 & 9 & 10 & 11 & 12 & 13 & 14 & 5 & 9 & 14 & 15 \\
\hline 1 & G. fossarum & - & 0.96 & 0.97 & 0.67 & 0.79 & 0.71 & 0.64 & 0.63 & 0.75 & 0.64 & 0.63 & 0.63 & 0.65 & 0.63 & 0.52 & 0.53 & 0.53 & 0.52 \\
\hline 2 & G. fossarum & 0.04 & - & 0.98 & 0.72 & 0.78 & 0.74 & 0.69 & 0.68 & 0.77 & 0.69 & 0.67 & 0.68 & 0.68 & 0.67 & 0.53 & 0.54 & 0.54 & 0.52 \\
\hline 3 & G. fossarum & 0.03 & 0.02 & - & 0.70 & 0.75 & 0.72 & 0.67 & 0.66 & 0.76 & 0.67 & 0.66 & 0.66 & 0.68 & 0.66 & 0.53 & 0.54 & 0.54 & 0.52 \\
\hline 4 & $G$. fossarum & 0.39 & 0.33 & 0.36 & - & 0.69 & 0.98 & 0.96 & 0.98 & 0.81 & 0.97 & 0.94 & 0.94 & 0.94 & 0.94 & 0.75 & 0.74 & 0.75 & 0.75 \\
\hline 5 & G. fossarum & 0.24 & 0.26 & 0.29 & 0.37 & - & 0.72 & 0.64 & 0.62 & 0.71 & 0.63 & 0.62 & 0.62 & 0.63 & 0.63 & 0.52 & 0.53 & 0.52 & 0.56 \\
\hline 6 & $G$. fossarum & 0.34 & 0.30 & 0.33 & 0.02 & 0.33 & - & 0.95 & 0.97 & 0.81 & 0.96 & 0.93 & 0.94 & 0.94 & 0.93 & 0.73 & 0.72 & 0.74 & 0.74 \\
\hline 7 & G. caparti & 0.44 & 0.37 & 0.39 & 0.04 & 0.44 & 0.05 & - & 0.98 & 0.79 & 0.97 & 0.96 & 0.93 & 0.93 & 0.93 & 0.74 & 0.74 & 0.74 & 0.73 \\
\hline 8 & G. caparti & 0.46 & 0.38 & 0.41 & 0.024 & 0.48 & 0.04 & 0.02 & - & 0.81 & 0.98 & 0.95 & 0.95 & 0.96 & 0.95 & 0.75 & 0.74 & 0.76 & 0.74 \\
\hline 9 & G. fossarum & 0.28 & 0.27 & 0.28 & 0.21 & 0.34 & 0.20 & 0.24 & 0.21 & - & 0.82 & 0.81 & 0.81 & 0.82 & 0.81 & 0.72 & 0.71 & 0.72 & 0.72 \\
\hline 10 & G. fossarum & 0.45 & 0.38 & 0.40 & 0.32 & 0.46 & 0.04 & 0.03 & 0.02 & 0.20 & - & 0.99 & 0.99 & 0.99 & 0.99 & 0.74 & 0.73 & 0.75 & 0.73 \\
\hline 11 & G. fossarum & 0.46 & 0.40 & 0.42 & 0.07 & 0.47 & 0.08 & 0.07 & 0.06 & 0.21 & 0.01 & 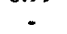 & 0.99 & 1,00 & 1,00 & 0.71 & 0.99 & 0.97 & 0.97 \\
\hline 12 & G. fossarum & 0.46 & 0.38 & 0.41 & 0.06 & 0.47 & 0.07 & 0.07 & 0.05 & 0.21 & 0.01 & 0.01 & - & 1,00 & 1,00 & 0.70 & 0.69 & 0.71 & 0.70 \\
\hline 13 & G. fossarum & 0.43 & 0.39 & 0.39 & 0.06 & 0.46 & 0.06 & 0.07 & 0.05 & 0.20 & 0.01 & 0.00 & 0.00 & - & 1,00 & 0.72 & 0.70 & 0.72 & 0.71 \\
\hline 14 & G. fossarum & 0.45 & 0.40 & 0.41 & 0.06 & 0.46 & 0.07 & 0.07 & 0.05 & 0.21 & 0.01 & 0.00 & 0.01 & 0.00 & - & 0.71 & 0.70 & 0.72 & 0.71 \\
\hline 5 & G.p. pulex & 0.65 & 0.63 & 0.64 & 0.29 & 0.66 & 0.32 & 0.31 & 0.29 & 0.33 & 0.30 & 0.34 & 0.36 & 0.33 & 0.34 & - & 0.99 & 0.97 & 0.97 \\
\hline 9 & G.p. pulex & 0.63 & 0.62 & 0.62 & 0.31 & 0.64 & 0.33 & 0.31 & 0.31 & 0.35 & 0.20 & 0.21 & 0.21 & 0.20 & 0.20 & 0.01 & - & 0.96 & 0.96 \\
\hline 14 & G.p. pulex & 0.64 & 0.62 & 0.63 & 0.28 & 0.64 & 0.30 & 0.30 & 0.28 & 0.32 & 0.29 & 0.33 & 0.35 & 0.32 & 0.33 & 0.33 & 0.04 & - & 0.98 \\
\hline 15 & G.p. pulex & 0.66 & 0.65 & 0.66 & 0.28 & 0.57 & 0.31 & 0.32 & 0.30 & 0.33 & 0.31 & 0.35 & 0.36 & 0.34 & 0.34 & 0.03 & 0.04 & 0.02 & 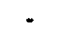 \\
\hline
\end{tabular}

\section{Genetic variation among populations}

Differences in electromorph distribution among the samples studied are shown in table 3. G. p. pulex populations are characterized by the predominating Est $-1^{a}, M p i^{d}$ and $P e p-2^{c}$ electromorphs. Except for G. fossarum from station 9 (table 1, 3; fig. 1A, C), the $P k^{b}$ electromorph is likewise restricted to populations of G. p. pulex.

The unique occurrence of the Got-2 ${ }^{d}$ and $H k-1^{a}$ electromorphs in $G$. fossarum samples from the Regensburg area (stations 1, 2 and 3; table 1, 3; fig. $1 \mathrm{~A}, \mathrm{D}$ and $\mathrm{E}$ ) discriminates them from nearly all other samples of the species studied.

The $M e^{b}$ electromorph was found in samples of G. fossarum trom the Regensburg area (stations 1, 2 and 3) and stations 9 and 5 (Belgium and Switzerland respectively; see Tables 1,3 and fig. $1 \mathrm{C}$ and F). The Got-1 $1^{a}$ electromorph is predominant in samples of $G$. fossarum from the Regensburg area and 
Table 6. Matrix of Rogers' (1972) distance.

\begin{tabular}{|c|c|c|c|c|c|c|c|c|c|c|c|c|c|c|c|c|c|c|c|}
\hline station no. & species & 1 & 2 & 3 & 4 & 5 & 6 & 7 & 8 & 9 & 10 & 11 & 12 & 13 & 14 & 5 & 9 & 14 & 15 \\
\hline 1 & G. fossarum & - & & & & & & & & & & & & & & & & & \\
\hline 2 & G. fossarum & 0.08 & - & & & & & & & & & & & & & & & & \\
\hline 3 & G. fossarum & 0.09 & 0.07 & - & & & & & & & & & & & & & & & \\
\hline 4 & G. fossarum & 0.34 & 0.31 & 0.34 & - & & & & & & & & & & & & & & \\
\hline 5 & G. fossarum & 0.24 & 0.26 & 0.30 & 0.34 & - & & & & & & & & & & & & & \\
\hline 6 & G. fossarum & 0.32 & 0.29 & 0.32 & 0.07 & 0.31 & - & & & & & & & & & & & & \\
\hline 7 & G. caparti & 0.38 & 0.34 & 0.36 & 0.09 & 0.37 & 0.11 & - & & & & & & & & & & & \\
\hline 8 & G. capari & 0.38 & 0.34 & 0.37 & 0.07 & 0.39 & 0.10 & 0.05 & - & & & & & & & & & & \\
\hline 9 & G. fossarum & 0.27 & 0.26 & 0.27 & 0.24 & 0.31 & 0.24 & 0.25 & 0.21 & - & & & & & & & & & \\
\hline 10 & G. fossarum & 0.37 & 0.33 & 0.36 & 0.09 & 0.38 & 0.10 & 0.07 & 0.04 & 0.20 & - & & & & & & & & \\
\hline 11 & G. fossarum & 0.38 & 0.35 & 0.36 & 0.12 & 0.38 & 0.13 & 0.10 & 0.07 & 0.21 & 0.04 & • & & & & & & & \\
\hline 12 & G. fossarum & 0.38 & 0.34 & 0.36 & 0.10 & 0.38 & 0.12 & 0.10 & 0.05 & 0.21 & 0.03 & 0.02 & - & & & & & & \\
\hline 13 & G. fossarum & 0.37 & 0.34 & 0.35 & 0.11 & 0.38 & 0.11 & 0.10 & 0.07 & 0.20 & 0.04 & 0.02 & 0.02 & - & & & & & \\
\hline 14 & G. fossarum & 0.38 & 0.35 & 0.36 & 0.12 & 0.38 & 0.13 & 0.11 & 0.07 & 0.21 & 0.03 & 0.01 & 0.03 & 0.02 & - & & & & \\
\hline 5 & G.p.pulex & 0.48 & 0.47 & 0.48 & 0.28 & 0.49 & 0.31 & 0.29 & 0.25 & 0.29 & 0.27 & 0.29 & 0.30 & 0.29 & 0.29 & - & & & \\
\hline 9 & G.p.pulex & 0.47 & 0.46 & 0.47 & 0.30 & 0.48 & 0.31 & 0.28 & 0.28 & 0.32 & 0.30 & 0.32 & 0.33 & 0.32 & 0.31 & 0.03 & - & & \\
\hline 14 & G.p.pulex & 0.47 & 0.46 & 0.47 & 0.27 & 0.48 & 0.30 & 0.28 & 0.25 & 0.29 & 0.27 & 0.29 & 0.30 & 0.29 & 0.29 & 0.04 & 0.07 & - & \\
\hline 15 & G.p.pulex & 0.49 & 0.48 & 0.49 & 0.27 & 0.45 & 0.29 & 0.30 & 0.28 & 0.31 & 0.29 & 0.31 & 0.32 & 0.31 & 0.30 & 0.06 & 0.08 & 0.05 & - \\
\hline
\end{tabular}

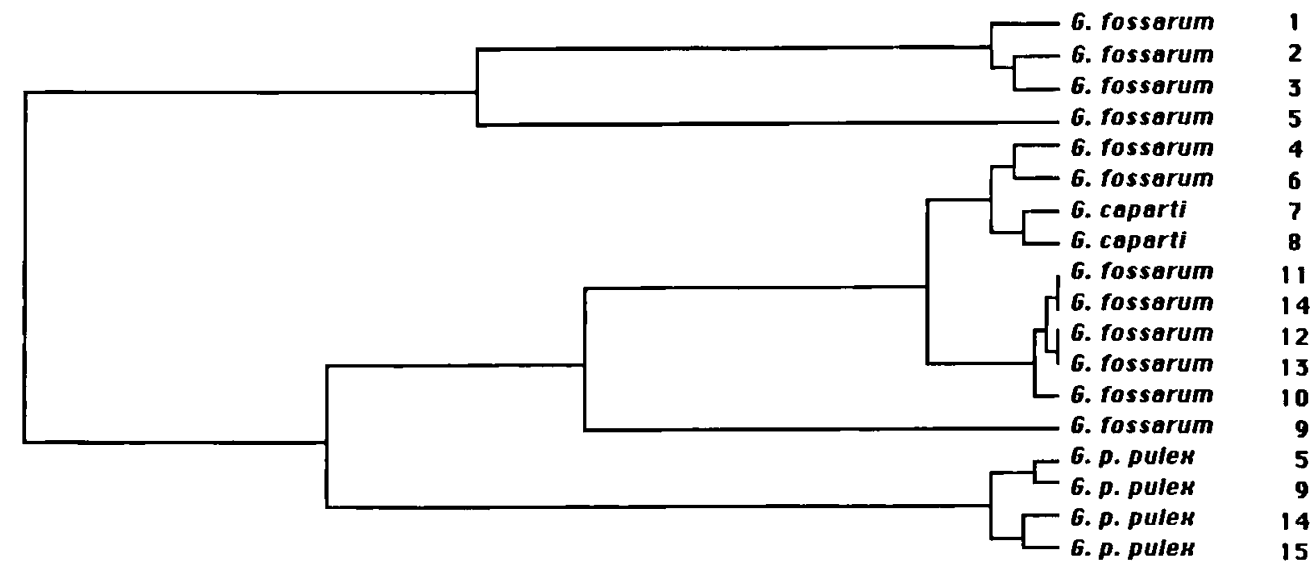

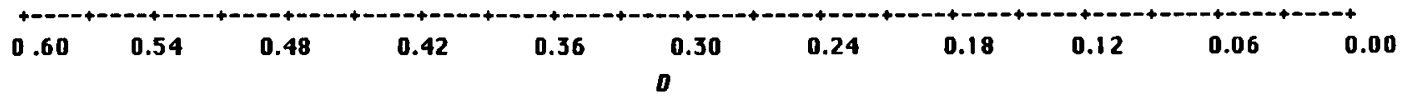

Fig. 3. UPGMA dendrogram of Nei's (1972) genetic distance $(D)$ based upon 20 enzyme loci.

station 5, but occurs also in the $G$. fossarum population from station 6 (table 1, fig. 1F).

A half matrix of diagnostic loci $(0.99$ criterion; Ayala \& Powell, 1972) is summarized in table 4. From the data in tables 5 and 6 , respectively, an UPGMA dendrogram and a distance Wagner network were generated (figs. 3 and 4).

The most conspicuous level of divergence is noted between samples of $G$. fossarum populations from the Regensburg area (stations 1, 2 and 3) plus station 5, and samples of the remaining stations; this level is even beyond the divergence level of $G$. pulex samples (stations 5, 9, 14 and 15; tables 1, 3, fig. 1A, B, C, E) with regard to samples of all the G. cf. fossarum populations involved (figs. 3, 4). Another cluster is formed by the samples of $G$. fossarum and $G$. caparti from the Channel coast (stations 10-14; tables 1, 3; fig. 1A and B), Switzer- 

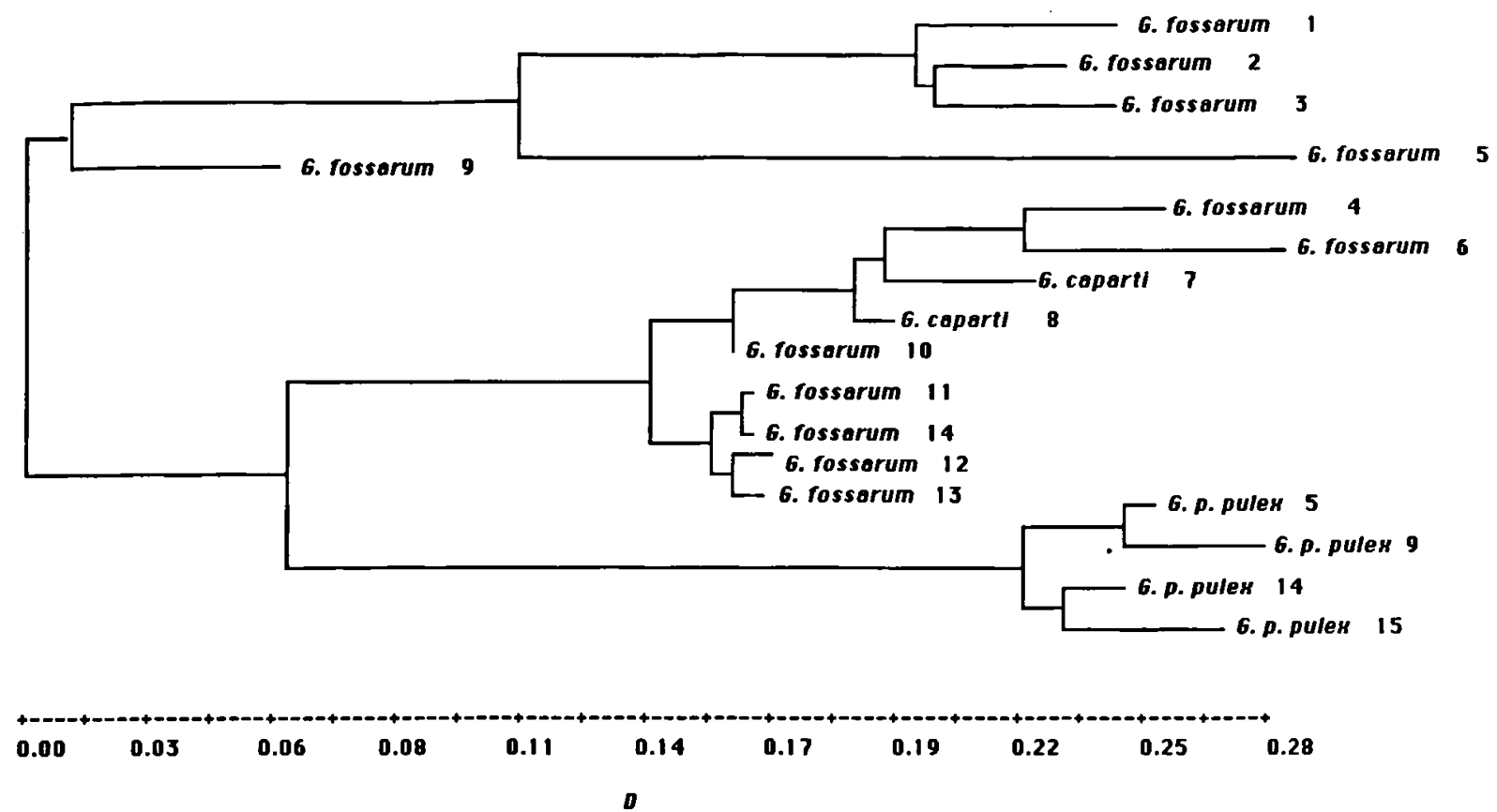

Fig. 4. Distance Wagner network of Rogers' (1972) genetic distance rooted at the midpoint of the longest path (Farris, 1972) based upon 20 enzyme loci.

Table 7. Mean intra- and inter-cluster values of the genetic identity ( $I ; \mathrm{Nei}, 1972)$, averaged over species, based upon 20 enzyme loci. Range $I_{\min }-I_{\max }$ in italics.

\begin{tabular}{|c|c|c|c|c|c|c|}
\hline Station no. & $\begin{array}{c}\text { G. fossarwm } \\
\begin{array}{c}4-11-12 \\
13-14\end{array} \\
\end{array}$ & $\begin{array}{c}\text { G. fossarum } \\
9\end{array}$ & $\begin{array}{c}\text { G. fossarum } \\
1-2-3\end{array}$ & $\begin{array}{c}\text { G. fossarwm } \\
5\end{array}$ & $\begin{array}{r}\text { G.p.pulex } \\
5.9 .14-15\end{array}$ & $\begin{array}{c}\text { G. caparni } \\
7-8\end{array}$ \\
\hline$\underset{13-14}{4-6-11-12}$ & $\begin{array}{c}0.97 \\
0.93 \cdot 1.00\end{array}$ & & & & & \\
\hline 9 & $\begin{array}{c}0.81 \\
0.81-0.82\end{array}$ & & & & & \\
\hline $1-2-3$ & $\begin{array}{c}0.67 \\
0.03-0.74\end{array}$ & $\begin{array}{c}0.76 \\
0.75-0.77\end{array}$ & $\begin{array}{c}0.97 \\
0.96-0.98\end{array}$ & & & \\
\hline 5 & $\begin{array}{c}0.65 \\
0.02-0.72\end{array}$ & $\begin{array}{c}0.71 \\
0.71-0.71\end{array}$ & $\begin{array}{c}0.77 \\
0.74-0.79\end{array}$ & & & \\
\hline $5-9-14-15$ & $\begin{array}{c}0.72 \\
0.69-0.75\end{array}$ & $\begin{array}{c}0.71 \\
0.70-0.72\end{array}$ & $\begin{array}{c}0.53 \\
0.52-0.54\end{array}$ & $\begin{array}{c}0.53 \\
0.51-0.56\end{array}$ & $\begin{array}{c}0.97 \\
0.96-0.99\end{array}$ & \\
\hline 7.8 & $\begin{array}{c}0.95 \\
0.93-0.98\end{array}$ & $\begin{array}{c}0.80 \\
0.79-0.81\end{array}$ & $\begin{array}{c}0.66 \\
0.63-0.69\end{array}$ & $\begin{array}{c}0.63 \\
0.62-0.64\end{array}$ & $\begin{array}{c}0.74 \\
0.73-0.76\end{array}$ & $\begin{array}{c}0.98 \\
0.98-0.98\end{array}$ \\
\hline
\end{tabular}

land (stations 4 and 6; tables 1, 3; fig. 1F), and Belgium (stations 7 and 8 ; tables 1, 3; fig. 1A, C). This cluster can be subdivided into two smaller clusters. The first one consists of the Swiss samples of $G$. fossarum from stations 4 and 6, and $G$. caparti from stations 7 and 8 . The second is formed by the genetically uniform samples of $G$. fossarum from the Channel coast area (stations 10-14). The $G$. fossarum samples from stations 5 (Switzerland; tables 1, 3; fig. 1F) and 9 (Belgium; tables 1, 3; fig. 1C) deviate genetically from the general pattern. From the data in table 5 mean inter- and intracluster values of $I$ can be calculated. These values (shown in table 7) suggest that there is no overlap of inter- and intra-cluster values of $I$, indicating (figs. 3, 4) stable clusters formed by: (a) G. fossarum from the Regensburg area (stations 1-3), (b) G. pulex (stations 5, 9, 14 and 15) and (c) G. fossarum $+G$. caparti from the Belgian stations 7 and 8 (G. caparti), the Swiss stations 4, 6, the French stations 11-14 and the Belgian station 10 (all $G$. fossarum). Intra-specific values of $I$ among $G$. fossarum samples from this cluster and inter-specific values of $I$ among samples of $G$. fossarum and $G$. caparti overlap almost entirely and do not differ significantly in a Mann-Whittney U-test $(U=98.5$; $p=0.48$ ). Thus, based on the allozyme data, samples attributed to $G$. caparti do not differ significantly from samples of $G$. fossarum in the same cluster (figs. 3, 4).

The topology of the UPGMA dendrogram (fig. 3) and the distance Wagner dendrogram (fig. 4) is almost the same. However, one conspicuous dif- 
ference is the position of the "deviating" samples of $G$. fossarum population from station 9 . In the distance Wagner network, this population clusters with $G$. fossarum samples from the Regensburg area (stations 1-3) and station 5 .

\section{Discussion and conclusions}

The genetic differentiation pattern of samples of the species examined (shown in figs. 3 and 4) is unexpected. Among the main clusters (a) (stations 1-3: G. fossarum), (b) (stations 5, 9, 14 and 15: $G$. p. pulex) and (c) (G. caparti from stations 7-8 and G. fossarum from stations 4,6 and 10-14) discussed above, $I$-values between cluster (b) and the presumed conspecific samples from cluster (c) range from $0.63-0.74$. Inter-specific values of $I$ between G. p. pulex (cluster b) and G. fossarum including $G$. caparti (clusters a and c) range from $0.52-0.54$ and $0.69-0.75$, respectively, which is of about the same order. With G. p. pulex (cluster b) as standard for genetic differentiation at the species level, the samples of $G$. fossarum examined warrant division into two different species: $G$. fossarum, including the samples from the Regensburg area (cluster b: stations 1-3) and possibly the samples from stations 5 and 9 (fig. 4); and a second species, including the samples from stations 4 and 6 , 10-14, and 7-8 (cluster c). According to rules of taxonomic nomenclature, the latter species should be named $G$. caparti Pètre-Stroobants, 1980.

The results of this study do not provide genetic evidence for recognition of $G$. caparti as a distinct species. Moreover, based upon the criteria of PètreStroobants (1980), only part of the individuals sampled at the type locality could be attributed to $G$. caparti, whereas others had to be attributed to $G$. fossarum (Pinkster, pers. comm.). Electrophoretically, however, the population sample seems perfectly homogeneous (table 3). Consequently, it must be concluded, that the samples of the Belgian stations 7 and 8 (G. caparti), the Swiss stations 4, 6, the French and Belgian stations 10-14 (all $G$. fossarum) are likely to be conspecific. Crossbreeding experiments between several morphologically distinct population samples of $G$. fossarum (including samples of $G$. caparti) yielded positive results (Goedmakers, 1972; Scheepmaker, unpubl. data). Most of these samples have been screened electrophoretically (Scheepmaker, unpubl. data) and proved to be genetically very similar. One could argue, that "true" $G$. fossarum population samples from the Regensburg area in the neighbourhood of the former type-locality (cluster b) were not involved in any of the cross-breeding experiments cited above. However, cross-breeding experiments involving species of Gammarus do not necessarily solve the taxonomic status among populations. For instance, in experiments by Scheepmaker et al. (1988), populations yielding $I$-values as low as 0.56 proved to be still partly interfertile.

In an attempt to avoid more confusion, rather than contributing to it, we would propose the term G. fossarum sensu lato for all populations hitherto identified as $G$. fossarum (including G. caparti), and reserve $G$. fossarum sensu stricto for the populations from the surroundings of its former typelocality in Regensburg (cluster b).

An explanation for the considerable amount of genetic divergence of $G$. fossarum s. str. with regard to the other population samples studied is probably provided by its distribution in the Danubian basin, which is characterized by a variety of species limited to this area (e.g. Thienemann, 1950). It is interesting to note that Siegismund (pers. comm.), who investigated $G$. cf. fossarum populations from brooks in the Rhine basin, did not find greater differences between these two areas than could be found within the Danube basin. However, these brooks in the Rhine basin were close to brooks belonging to the Danube drainage system.

In fig. 3, Swiss and Belgian populations samples of $G$. fossarum s.l. belonging to the Rhine- and Meuse drainage systems (stations 4 and 6-8) are differentiated from the Channel coast population samples (stations 10-14). These 2 subclusters may have a common descent from an ancestor inhabiting the upper reaches of streams of unglaciated lowlands during one or more glaciations. Differentiation may have originated by populations following the withdrawing ice. This hypothesis seems in agreement with the configuration of $G$. fossarum 
s.l. populations from stations $10,8,7$ and 4 , forming a monophyletic group in fig. 4.

The Swiss stations 4-6 are situated in the same, rather limited geographical area (fig. 1A, F). According to Thienemann (1950), stations 4 and 6 are located in a part of this area covered with ice during the Würm glacial. Station 5, however, is located in a tongue of land beyond the maximum extension of the Würm glaciers. This circumstance might explain the deviating character of the $G$. fossarum s.l. population sample from station 5 . This population possibly represents the original form, whereas the area once covered with ice was recolonized by populations from a different ancestor. An explanation for the other "deviating" population, G. fossarum s.l. from station 9 , is not readily at hand.

The genetic differentiation of geographically distant population samples of $G$. p. pulex is homogeneous with reference to G. fossarum s.l. (figs. 3, 4). This is probably because G. p. pulex inhabits middle and lower reaches of rivers, allowing genetic exchange among drainage systems, whereas $G$. fossarum s.l. generally inhabits the upper reaches. As the upper reaches are generally separated from each other by lower reaches, G. fossarum s.l. populations are therefore more subjected to genetic isolation.

Although there is a considerable overlap in morphological variation among the populations studied, the genetic variation recorded in $G$. fossarum s.l. seems to be paralleled by some morphological differentiation (table 2). For instance, all population samples from the Channel coast area (stations 10-14; fig. 1A, B), forming a cluster in fig. 3, are characterized by a short setation of the A2 and P3; individuals from Swiss populations all have relatively long setae on the flagellum of the A2; individuals from station 1-3 do not always bear calceoli; individuals from the "deviating" population 9 differ from all other population samples by the longer setation of the peduncle segment 5 of $\mathrm{A} 2$, and the short setation of P3, etc. (table 2). However, one should be extremely careful: none of these characters are singly diagnostic, and only particular combinations of characters may designate a group of populations. Moreover, many of these charac- ters are subject to allometric growth and seasonal variation.

\section{Acknowledgements}

We wish to thank Drs. C.B. Crabtree, S.B.J. Menken, S. Pinkster, H.R. Siegismund and Prof. Dr. J.H. Stock for commenting on the manuscript.

\section{References}

Ayala, F.J. \& J.R. Powell, 1972. Allozymes as diagnostic characters of sibling species of Drosophila. Proc. natn. Acad. Sci. USA, 69(5): 1094-1096.

Farris, J.S., 1972. Estimating phylogenetic trees from distance matrices. Am. Natural., 106: 645-668.

Goedmakers, A., 1972. Gammarus fossarum Koch, 1835: Redescription based on neotype material and notes on its local variation (Crustacea, Amphipoda). Bijdr. Dierk., 42(2): 124-138.

Karaman, G.S. \& S. Pinkster, 1977. Freshwater Gammarus species from Europe, North Africa and adjacent regions of Asia (Crustacea, Amphipoda). Part 1. Gammarus pulex group and related species. Bijdr. Dierk., 47 (1): 1-97.

Meijering, M.P., 1972. Physiologische Beiträge zur Frage der systematischen Stelling von Gammarus pulex und Gammarus fossarum Koch. Crustaceana, Suppl. 3: 313-325.

Nei, M., 1972. Genetic distance between populations. Am. Natural., 106: 283-292.

Pètre-Stroobants, G., 1980. Diagnose préliminaire de Gammarus caparti, une nouvelle espèce de Gammarus de Belgique (Crustacea, Amphipoda). Bull. Ann. Soc. r. belge Ent., 116: 135.

Pètre-Stroobants, G., 1981. Une nouvelle espèce de Gammarus en Belgique: Gammarus caparti n. sp. (Crustacea, Amphipoda). Bull. Ann. Soc. r. Sci. nat. Belg., 53(6): 1-6.

Pinkster, S., 1983. The value of morphological characters in the taxonomy of Gammarus. Beaufortia, 33(2): 15-28.

Rogers, J.S., 1972. Measures of genetic similarity and genetic distance. Studies in Genetics, Univ. Texas Publ., 7213: 145-153.

Roux, A.L., 1971. Les Gammares du groupe pulex. III. Essais d'hybridation et examen caryologique. Arch. Zool. exp. gén., 112: 819-868.

Scheepmaker, M., 1987. Morphological and genetic differentiation of Gammarus stupendus Pinkster, 1983 in the Massif de la Sainte Baume, France. Bijdr. Dierk., 57(1): 1-18.

Scheepmaker, M., F. van der Meer \& S. Pinkster, 1988. Genetic differentiation of the Iberian amphipods Gammarus ibericus Margalef, 1951 and G. gauthieri S. Karaman, 1935, with reference to some related species in France. Bijdr. Dierk., 58(2): 205-226. 
Sneath, P.H.A. \& R.R. Sokal, 1973. Numerical taxonomy: i-xv, 1-780. (W.H. Freeman and Company, San Francisco). Swofford, D.L. \& R.B. Selander, 1981. Biosys-1. A computer program for the analysis of allelic variation in genetics. (Dept. of Genetics and Development, Univ. of Illinois at UrbanaChampaign, Urbana, Illinois.)

Thienemann, A., 1950. Verbreitungsgeschichte der Süsswasser- tierwelt Europas. Binnengewässer, Stuttgart, 18: [i-xiv], 1-809.

Wautier, J. \& A.L. Roux, 1959. Note sur les Gammares du groupe pulex dans la région Lyonnaise. Bull. mens. Soc. Linn. Lyon, 28(3): 76-83.

Received: 3 April 1989 